\title{
Aging on the Input Versus Output Side: Theoretical Implications of Age-Linked Asymmetries Between Detecting Versus Retrieving Orthographic Information
}

\author{
Donald G. MacKay, Lise Abrams, and Manissa J. Pedroza \\ University of California, Los Angeles
}

\begin{abstract}
This experiment tested for age-linked asymmetries predicted under Node Structure theory (NST; D. G. MacKay \& D. M. Burke, 1990) between detecting versus retrieving orthographic information. Older adults detected that briefly presented words were correctly spelled (e.g., endeavor) or misspelled (e.g., endeavuor) as readily as did young adults. However, they were less able than young adults to retrieve the correctly and incorrectly spelled words that they had seen. These age-linked asymmetries were not due to educational factors, stimulus characteristics, sensory-level factors, task complexity, floor or ceiling effects, general slowing, or cohort-related activities, but they were consistent with NST predictions and with similar asymmetries in a wide range of other studies. By contrast, repetition deficits in detecting and retrieving repeated- versus unrepeated-letter misspellings (e.g., elderdly vs. elderkly) were symmetrical or equivalent in magnitude for young and older adults. Implications for a wide range of theories of cognitive aging and of repetition deficits are discussed.
\end{abstract}

In this article we report the first systematic comparison of aging effects on the input versus output side of language processing. The input side of language involves perception of the letters and speech sounds that make up words as well as comprehension of the meaning of words and sentences. The output side of language involves retrieval of words and speech sounds during speech production and retrieval of letters in words during writing and typing. We first review the many studies that have examined effects of aging separately on either the input or output side of language and argue that age effects in these studies are asymmetrical: When age-linked deficits in sensory processing and in the ability to encode new information are controlled or factored out, input-side processes remain remarkabiy stable in old age, whereas output-side processes exhibit major declines. We then argue that this asymmetrical pattern, if not attributable to experimental artifact, presents a fundamental challenge for theories of cognitive aging, which must explain why some aspects of information processing are so much more vulnerable to the effects of aging than others: Theories that predict either sparing or impairment of verbal abilities across the board in old age do not fit this asymmetrical pattern. Finally, we describe a theory that predicts two specific types of input-output asymmetry and present our experiment, in which we

Donald G. MacKay, Lise Abrams, and Manissa J. Pedroza, Department of Psychology, University of California, Los Angeles.

Lise Abrams is now at the Department of Psychology, University of Florida.

This research was supported by National Institute on Aging Grant R01 AG 097705 and a National Science Foundation graduate fellowship to Lise Abrams. We thank Deborah Burke, Larry Hochhaus, Lori James, Susan Kemper, and Michelle Miller for helpful comments on a draft of this article and Bryan Chae and Victor Hwang for research assistance.

Correspondence concerning this article should be addressed to Donald G. MacKay, Department of Psychology, University of California, Los Angeles, California 90095-1563. Electronic mail may be sent to mackay@ psych.ucla.edu. tested these predictions and controlled for possible artifacts in previous studies.

\section{Language and Aging on the Input Side}

The semantic priming paradigm has provided many dramatic demonstrations of age constancy on the input side: Laver and Burke (1993) reviewed the results of 15 semantic priming studies indicating that presenting a word (e.g., doctor) causes the automatic spread of activation to related words (e.g., nurse) without a deficit in old age (for more recent reviews, see Burke \& MacKay, 1997; MacKay \& Abrams, 1996). Studies of word comprehension in sentences have shown similar age constancy using off-line measures (for a recent review, see Burke \& MacKay, 1997) and on-line measures of semantic processes as they occur during sentence comprehension (see, e.g., Burke \& Yee, 1984; G. Cohen \& Faulkner, 1983; Light, Valençia-Laver, \& Zavis, 1991; Madden, 1988; Stine \& Wingfield, 1994; Wingfield, Alexander, \& Cavigelli, 1994). These "on-line" data ruled out a major confound inherent in "off-line," or recall-based, measures of comprehension, in which age differences may reflect not word comprehension per se but the process of forming new connections for linking comprehended words to their temporal and spatial context or source (for a review, see Burke \& MacKay, 1997).

\section{Language and Aging on the Output Side}

Unlike language comprehension, language production is not immune to the effects of aging: Spoken and written language production exhibit large age-related declines that are independent of the ability to encode new information (for recent reviews, see Burke \& MacKay, 1997; MacKay \& Abrams, 1998). Older adults often complain of increased difficulty in retrieving words that they know they know (see, e.g., Burke, MacKay, Worthley, \& Wade, 1991), and their performance on a range of tasks is consistent with 
these complaints. For example, older adults in the tip-of-thetongue state can report fewer phonological features of a target word, such as its stress pattern and initial letter, and have fewer phonologically similar words come spontaneously to mind than do young adults (see, e.g., Rastle \& Burke, 1996). These and other age-linked problems in spoken word retrieval (see, e.g., Au et al,, 1995) have a close link to the access of phonological information because providing phonological cues to a target word reduces the age deficits (see, e.g., Mitchell, 1989), but providing additional semantic information does not (Bowles \& Poon, 1985).

Retrieval of orthographic information also exhibits age-linked declines. Young (aged 17-23 years), older (aged 60-71 years), and very old adults (aged 73-88 years) in MacKay and Abrams (1998) heard irregularly spelled English words spoken slowly, clearly, and repeatedly and wrote the words down at their own pace on a sheet of paper. Despite having more education and higher vocabulary scores, the oldest adults correctly spelled high-frequency words less often than did the young adults, an age-linked decline that was unrelated to the rated quality of previous training on spelling skills; to hours per week spent reading, writing, or solving crossword puzzles; and to age-linked declines in the ability to detect and correct errors in their written output. Furthermore, responses to a metamemory questionnaire indicated that the oldest but not the older adults were aware of their declining ability to spell.

\section{Theories of Aging on the Input Versus Output Side}

What explains the asymmetrical effects of aging in these separate studies of perception and production? One possibility is experimental artifact: The participants and the stimuli differed in the studies on perception versus production, and the perception tasks may have been "easier" than the production tasks. Such "artifact accounts" are consistent with the hypothesis that the effects of aging on perception and production are symmetrical or equivalent in magnitude. This symmetry hypothesis derives from theories that postulate mirror-image perception and production processes and attribute cognitive aging to a single factor such as general slowing.

The asymmetry hypothesis, which states that aging has disproportionately greater effects on production than perception, derives from theories in which production processes are not the mirror image of perception processes and in which cognitive aging impairs production relatively more than perception. An example is the Transmission Deficit hypothesis (TDH) as embedded within the Node Structure theory (NST) of MacKay (1987). Because we tested several age-linked asymmetries between detecting versus retrieving orthographic information predicted by this theory, we examine its mechanisms in detail.

Because MacKay and Abrams (1998) described the top-down processes underlying orthographic retrieval in NST, we do not reiterate them here. Instead, we elaborate on the bottom-up processes for orthographic detection and how these bottom-up processes differ from the top-down processes for retrieval. For illustration, consider the letter nodes in Figure 1, which represent the orthographic string aging ${ }^{1}$ during both detection and retrieval in NST. For detection, these letter nodes are connected bottom-up to a single lexical node that represents the concept of aging (see Figure 1); for retrieval, the lexical concept node for aging is connected top-down to these same letter nodes and to the systems

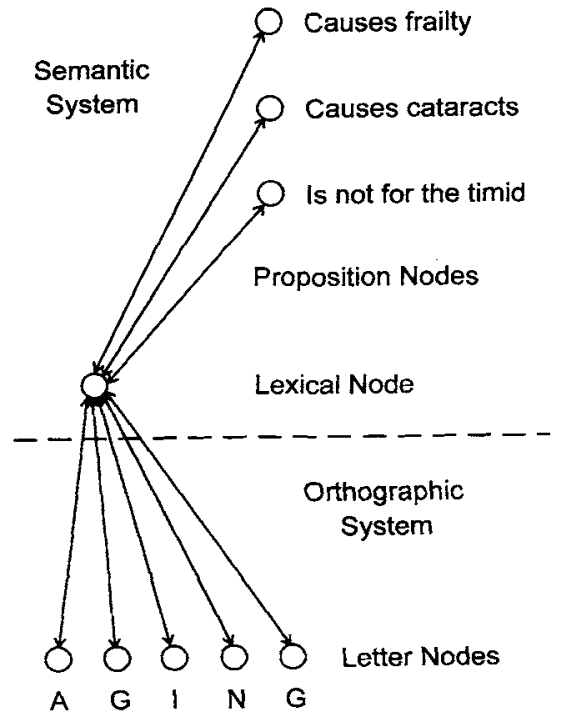

Figure 1. Some of the bidirectional connections in Node Structure theory for perceiving and producing the visually presented word aging. Also shown is a subset of proposition nodes representing semantic knowledge associated with the concept aging.

of muscle movement nodes for writing and typing the word aging (not shown in Figure 1). What is important to note in Figure 1 is that top-down and bottom-up connections are asymmetrical: The bottom-up links for perceiving the word aging converge many to one onto the lexical node for the word aging, whereas the topdown links for producing the word aging are one to one (i.e., only one node primes each letter node).

According to the TDH (MacKay \& Burke, 1990), aging causes a statistical decrease in the transmission of priming across connections between millions of nodes throughout the entire network. This means that age effects will be greatest when a node critical to a task receives priming from only a single source or connection within the network: If that one connection is defective, the critical node will be difficult to activate because no other sources of priming can offset the reduced priming across that connection. For example, a transmission deficit across the top-down link between the lexical concept and the letter node for $I$ in Figure 1 would make $I$ difficult to retrieve when writing or typing the word aging because no other source of priming could offset the transmission deficit. However, when perceiving the visual word aging, all five bottom-up connections from its letter nodes simultaneously deliver priming to the lexical node for aging. Because the resulting summation of priming across these connections will tend to offset an age-linked transmission deficit across any one link, the word aging will be accurately perceived despite such a transmission deficit. The TDH therefore predicts small or nonexistent age deficits in perceiving orthography despite large age deficits in producing or retrieving orthography.

\footnotetext{
${ }^{1}$ Figure 1 simplifies English orthographic representations for purposes of illustration. For example, the "letter nodes" in Figure 1 represent both letters and letter locations. Also, a full representation would include a hierarchically organized unit to represent the common English morpheme [-ing].
} 
The TDH also explains semantic priming effects. The semantic system consists of a vast number of lexical nodes and propositions that are either directly or indirectly interconnected with one another. Figure 1 illustrates a simplified semantic memory consisting of three propositions: Aging causes frailty, aging causes cataracts, and aging is not for the timid. These three propositions are interconnected via their common links to the lexical node for aging, which will also have indirect links to numerous other concepts via the many other propositions stored in semantic memory. For example, if the semantic memory in Figure 1 also contains the proposition cataracts cause blindness, then the concepts aging and blindness will have an indirect link via the propositions aging causes cataracts and cataracts cause blindness. Because of these indirect links between aging and blindness, activating the concept aging in the semantic priming paradigm will prime the concept blindness, enabling faster responses to this semantically related word.

Consider now the phenomenon of age constancy in semantic priming tasks. Semantically related concepts such as aging and blindness are more likely to have indirect links for older than young adults because over the course of their (longer) lifetimes, older adults have acquired more general knowledge in the form of propositions such as aging can cause glaucoma and glaucoma can cause blindness. As a result of this enriched semantic network, more "parallel" connections will link semantically related concepts in the memory systems of older than young adults. Priming will therefore converge across these additional, parallel connections and summate to a greater extent for older adults, thereby offsetting transmission deficits across any one connection. As a result, semantic priming effects will be as large or larger for older adults relative to young adults, as Laver and Burke (1993) observed.

\section{Our Paradigm: Detection Versus Retrieval of Orthographic Information}

To address the issue of artifacts in previous studies of perception versus production, we used exactly the same participants and stimuli in perception and production tasks that were comparable in difficulty, as measured post hoc. Participants saw briefly presented words that were either correctly spelled or deliberately misspelled, sometimes by adding a single "target" letter. For example, $U$ was the target letter added to endeavor in the misspelling endeavuor (the target letter is underlined), and these target letters were the focus of many of our analyses. Participants were instructed that the words they would see were either correctly spelled or misspelled (in unspecified ways) and that they had two tasks. The first was a perception task, in which they had to respond "right" to indicate a correct spelling or "wrong" to indicate an error detection. The second task, production and retrieval, was to write out the spelling of the word exactly as presented.

The symmetry hypothesis predicts no age-linked asymmetries between the detection versus retrieval tasks (i.e., no difference in effects of aging on detecting vs. retrieving misspelled and correctly spelled words). However, the asymmetry hypothesis predicts two age-linked asymmetries for the detection versus retrieval tasks: a correct spelling asymmetry (i.e., a smaller age-linked deficit in detecting than in retrieving the correct spelling of words that were recognized as correctly spelled) and a misspelling asymmetry (i.e., a smaller age-linked deficit in detecting misspelled stimuli than in retrieving misspelled stimuli given error detection).

We also adopted the logic of "double dissociation" procedures in neuropsychology (see Parkin, 1996, pp. 9-11) to test whether the misspelling asymmetry is specific to aging or whether it is more general in nature, such that any factor that influences the retrieval of misspellings exhibits the same interaction with aging or with task as predicted for the misspelling asymmetry. As the first step in this double dissociation logic, we introduced into a subset of our materials a variable with known effects on the retrieval of misspellings: repetition. That is, the added letter created either a repeated-or unrepeated-letter misspelling of these words. In repeated-letter misspellings the added or "target" letter repeated a "pretarget" letter earlier in the word (e.g., elderdly [target letter underlined and pretarget in italics], whereas in unrepeated-letter misspellings the target letter differed from all other letters in the word (e.g., elderkly). On the basis of previous results for young adults in a related paradigm (MacKay, 1969), we predicted a repetition deficit (RD) in perception and retrieval (i.e., better detection and retrieval of unrepeated- than repeated-letter misspellings). This being the case, the double dissociation logic proceeds as follows: If RD is greater in retrieval than in perception, especially for older adults, this would indicate that the misspelling asymmetry is general in nature and does not require an explanation within theories of cognitive aging. On the other hand, if RD is equivalent in retrieval and perception for both young and older adults, this would indicate that the misspelling asymmetry is specific to aging and requires an explanation within theories of cognitive aging.

The effects of aging on RD were also of interest for subsidiary reasons: MacKay, Miller, and Schuster (1994) and MacKay and Miller (1996) demonstrated that interactions between aging and word-level repetition can directly test four general hypotheses as to the causal basis of RD in the standard RD paradigm (see, e.g., Kanwisher \& Potter, 1990; MacKay \& Miller, 1994), in which words are presented one on top of the other using the rapid serial visual presentation (RSVP) technique. In the Appendix we outline age effects predicted for our paradigm under these four general RD hypotheses; how these predictions relate to our results and to familiar age-linked effects such as binding deficits (see, e.g., Burke \& MacKay, 1997, for a review) and inhibition deficits (see, e.g., McDowd, Oseas-Kreger, \& Filion, 1995); and the pros and cons of our paradigm versus the standard paradigm for testing these RD hypotheses. Table 1 contains the full set of hypotheses and predictions.

\section{Method \\ Participants}

The 72 participants fell into young and older groups. The means and standard deviations for standard background characteristics are shown in Table 2. All were native English speakers who reported no neurological problems, no serious medical problems, and normal or corrected-to-normal vision. The 36 young adults (14 men and 22 women) were aged 17-23 years and participated in return for course credit from introductory psychology classes at the University of California, Los Angeles (UCLA). The 36 older adults ( 17 men and 19 women) were aged $62-85$ years and were members of the UCLA Cognition and Aging Lab pool (current sample size $=198$ ) who received $\$ 10$ plus travel expenses from their 
Table 1

Summary of Primary and Secondary (RD) Hypotheses, Their Predictions, and Their Relation to the Present Results

\begin{tabular}{|c|c|c|}
\hline Hypothesis & Predictions & Outcome \\
\hline \multicolumn{3}{|l|}{ Primary } \\
\hline Asymmetry hypothesis & $\begin{array}{l}\text { Disproportionately greater effects of aging on } \\
\text { production than perception of both correct and } \\
\text { incorrect spelling }\end{array}$ & Supported \\
\hline Symmetry hypothesis & $\begin{array}{l}\text { Equivalent effects of aging on production and } \\
\text { perception of both correct and incorrect spelling }\end{array}$ & Unsupported \\
\hline \multicolumn{3}{|l|}{ Secondary } \\
\hline Basic RD hypotheses & $\begin{array}{l}\text { Greater detection and retrieval of unrepeated- than } \\
\text { repeated-letter misspellings (double dissociation } \\
\text { preconditions) }\end{array}$ & Supported \\
\hline Inhibition RD hypothesis & $\begin{array}{l}\text { More RD for young than older adults in detection, more } \\
\text { RD for young than older adults in reproducing } \\
\text { detected misspellings }\end{array}$ & Unsupported \\
\hline Binding RD hypothesis & $\begin{array}{l}\text { More RD for older than young adults in detecting } \\
\text { misspellings and in reproducing detected } \\
\text { misspellings, greater positional uncertainty in } \\
\text { retrieval of repeated than unrepeated letters, } \\
\text { especially for older adults }\end{array}$ & Unsupported \\
\hline $\begin{array}{l}\text { Refractory period RD } \\
\text { hypothesis }\end{array}$ & $\begin{array}{l}\text { An Age } \times \text { Repetition } \times \text { Presentation Rate interaction, } \\
\text { with more } \mathrm{RD} \text { for older than young adults at } \\
\text { moderate rates but equivalent RD at fast and at slow } \\
\text { rates }\end{array}$ & Unsupported \\
\hline Fusion RD hypothesis & Same as for refractory period $\mathrm{RD}$ hypothesis & Unsupported \\
\hline
\end{tabular}

Note. $\mathrm{RD}=$ repetition deficit.

homes in the greater Los Angeles area. Most were recruited from the UCLA alumni association, local churches, and senior citizen centers. The age groups differed at the .05 level on only two background characteristics: Older adults had more years of education than young adults, $t(68)=6.14$, $p<.001$, and higher Nelson-Denny Vocabulary Scale scores than did young adults, $t(68)=5.88, p<.001^{2}$

\section{Materials}

The materials consisted of 100 words that fell into two categories: Type 1 words $(n=45)$ and Type 2 words $(n=55)$. Type 1 words were selected from Faucett and Maki (1932), had a relatively high frequency of occurrence (mean $=80.4$ per million; Francis \& Kucera, 1982), and were 8.3 letters long on average (range $=7-10$ letters). Besides testing for asymmetries, Type 1 words were designed to assess the effects of repetition on the detection and retrieval of misspelings. Each Type 1 word appeared in three different spelling conditions counterbalanced across participants: correct spelling (e.g., elderly), unrepeated-letter misspelling (e.g., elderkly), and repeated-letter misspelling

Table 2

Background Characteristics of Participants

\begin{tabular}{|c|c|c|c|c|}
\hline \multirow[b]{2}{*}{ Variable } & \multicolumn{2}{|c|}{ Young adults } & \multicolumn{2}{|c|}{ Older adults } \\
\hline & $M$ & $S D$ & $M$ & $S D$ \\
\hline $\operatorname{Age}^{\mathrm{a}}$ & 19.03 & 1.47 & 70.33 & 5.39 \\
\hline Years of education ${ }^{2}$ & 13.03 & 1.43 & 16.30 & 2.32 \\
\hline Health rating & 7.17 & 2.18 & 8.13 & 1.76 \\
\hline Nelson-Denny Vocabulary ${ }^{2}$ & 14.68 & 3.47 & 20.79 & 3.07 \\
\hline Digit recall: Forward & 7.45 & 1.24 & 7.23 & 1.33 \\
\hline Digit recall: Backward & 5.24 & 1.22 & 5.35 & 1.29 \\
\hline
\end{tabular}

${ }^{\text {a }}$ Differences between age groups significant at the .05 level or less. (e.g., elderdly). As in these examples, Type 1 words were misspelled by adding a single target letter to the original word and always differed in pronunciation from the original word. In unrepeated-letter misspellings (e.g., elderkly) the added target letter differed from all other letters in the original word, whereas in repeated-letter misspellings the target letter repeated a letter earlier in the original word (e.g., elderdly). Target letters always had identical height characteristics in repeated- and unrepeated-letter misspellings and always occupied the same position within the original word, as in elderkly and elderdly, and they always resulted in misspellings that were phonologically and orthographically acceptable in English. Pretarget letters were the identical letter that preceded the added letter in repeated-letter misspellings (e.g., the initial $d$ in elderdly) and the corresponding letter in unrepeated-letter misspellings (e.g., the $d$ in elderkly). Pretarget letters never began a misspelled word and preceded target letters by one intervening letter (e.g., faculty misspelled as facultly), two intervening letters (e.g., reverence misspelled as revervence), or three intervening letters (e.g., bachelor misspelled as bachealor).

Type 2 words were similar to Type 1 words, except that they came from Norback (1974), were difficult to spell, never involved the letter repetition factor, and appeared only in one correctly spelled version $(n=35)$ or incorrectly-spelled version ( $n=20)$ ranging in length from 5 to 12 letters. Type 2 misspellings were formed by either replacing a single letter in the original word (e.g., quadrant misspelled as quadrent) or omitting a single letter (e.g., handkerchief misspelled as hankerchief). Type 2 words were designed to prevent participants from developing strategies attuned to the

\footnotetext{
${ }^{2}$ These degrees of freedom reflect the fact that we failed to obtain Nelson-Denny data from 2 young participants and education data from 2 older participants. In addition, 6 older participants did not indicate how many hours per week they spent solving crossword puzzles in the aboutto-be described background questionnaire. Finally, we dropped the correctly spelled word judgment from our about-to-be described Type 2 stimuli because it turned out to have two viable spellings.
} 
repetition factor and to further test for age-linked asymmetries independent of the repetition factor.

\section{Choice of Presentation Times}

We chose three overlapping presentation times for young and older adults on the basis of two sets of criteria. One set was designed to equate the relative difficulty of the perception and production tasks: We adjusted presentation rates until the performance of young pilot participants was about equal in the two tasks for both correctly and incorrectly spelled stimuli. The second set of criteria involved procedures developed in MacKay et al. (1994) to avoid ceiling and floor effects as well as possible confounds between RD, aging, and performance levels for unrepeatedminus repeated-letter targets. Using these criteria, we used $290 \mathrm{~ms} /$ word for both groups, enabling standardized between-groups comparisons, and the remaining rates were 230 and $260 \mathrm{~ms} /$ word for young adults and 340 and $390 \mathrm{~ms} /$ word for older adults. These rates could be considered fast, medium, and slow for young and older adults, with performance that was above a predetermined floor $(30 \%)$ and below a predetermined ceiling $(90 \%)$ for both groups.

\section{Procedure}

Participants first provided background information and then completed a questionnaire on activities with possible relations to spelling ability: hours per week spent reading, writing, and solving crossword puzzles. Participants also used 5-point scales to evaluate their current spelling ability and to rate how rigorously their grade school taught spelling skills $(5=$ rigorous training, $1=$ lax training). Questionnaires for the older adults also contained an identical 5-point scale for rating their remembered spelling ability at age 20 . Participants then saw six practice stimuli, two at each rate, with one word spelled correctly and five misspelled by repeating, adding, omitting, and replacing a letter. Written instructions informed participants that words would appear briefly for varying durations, centered on the screen before them, with some words spelled correctly and others incorrectly.

In the experiment proper, participants saw the 45 Type 1 words interspersed among the 55 Type 2 words, which occurred in three differing random orders across participants. Type 1 words appeared equally often in the three speliing and rate conditions, crossed over nine different random orders that were assigned to participants in each group by order of arrival. Type 2 words also appeared equally often in the three rate conditions across the nine versions of the experiment. However, to maintain relatively high attention levels and success rates over the experiment, the experimenter verbally informed participants about the upcoming word for all Type 2 words (e.g., "the next correctly or incorrectly spelled word is quadrant" for the misspelled stimulus quadrent).

Materials were presented via a Mac IIci computer using PsyScope software (J. D. Cohen, MacWhinney, Flatt, \& Provost, 1993), beginning with a 2,000-ms warning ("Get ready for the next word"), followed by a 1,200-ms fixation point before word presentation. Next came a string of 14 question marks (?????????????), signaling participants to say "right" (indicating that the word was correctly spelled) or "wrong" (otherwise). Participants next printed exactly what they had seen on a numbered sheet, (mis)spelling the word as they had seen it (mis)spelled, guessing at the spelling if necessary. The experimenter pressed any key to initiate the next trial.

\section{Results}

Table 1 shows our primary and secondary hypotheses and how they relate to our results. In the Main Results section, we include analyses relevant to the correct spelling and misspelling asymmetries discussed in the introduction. Subsidiary results include analyses of repetition and rate related to age-linked asymmetries. In the Appendix we include results related to our secondary hypotheses on the age-linked effects of repetition and the causal basis of RD. Unless indicated otherwise, all analyses and figures involved Type 1 stimuli; data for Type 2 stimuli are presented only in tables.

\section{Main Results}

Our simplest dependent measures were detection probability (i.e., the probability of correctly responding "right" or "wrong" in the detection task) and retrieval probability (i.e., the probability of correctly reproducing the stimuli exactly as presented, with each stimulus letter in corresponding order in the written record). Table 3 shows the mean detection probabilities per participant as a function of age, rate, and spelling condition (correctly spelled, repeated-, and unrepeated-letter misspellings). Table 4 shows the mean retrieval probabilities as a function of age, rate, and spelling condition.

Table 3

Probability of Correct Detection as a Function of Age, Rate, and Spelling Condition for Type 1 Words

\begin{tabular}{|c|c|c|c|c|c|c|c|c|c|c|}
\hline \multirow[b]{3}{*}{ Age group/Spelling condition } & \multicolumn{10}{|c|}{ Rate of presentation (ms/word) } \\
\hline & \multicolumn{2}{|c|}{230} & \multicolumn{2}{|c|}{260} & \multicolumn{2}{|c|}{290} & \multicolumn{2}{|c|}{340} & \multicolumn{2}{|c|}{390} \\
\hline & $M$ & $S D$ & $M$ & $S D$ & $M$ & $S D$ & $M$ & $S D$ & $M$ & SD \\
\hline \multicolumn{11}{|l|}{ Young adults } \\
\hline Correct spelling & .922 & .129 & .861 & .178 & .922 & .138 & & & & \\
\hline Unrepeated-letter misspellings & .843 & .215 & .893 & .132 & .900 & .141 & & & & \\
\hline Repeated-letter misspellings & .494 & .251 & .406 & .246 & .544 & .251 & & & & \\
\hline Repetition deficit ${ }^{2}$ & .349 & & .487 & & .356 & & & & & \\
\hline \multicolumn{11}{|l|}{ Older adults } \\
\hline Correct spelling & & & & & .928 & .119 & .922 & .138 & .915 & .122 \\
\hline Unrepeated-letter misspellings & & & & & .839 & .268 & .835 & .201 & .860 & .208 \\
\hline Repeated-letter misspellings & & & & & .472 & .310 & .528 & .314 & .528 & .291 \\
\hline Repetition deficit ${ }^{a}$ & & & & & .367 & & .394 & & .387 & \\
\hline
\end{tabular}

${ }^{a}$ Repetition deficits are the unrepeated condition minus the repeated condition. 
Table 4

Probability of Correct Retrieval as a Function of Age, Rate, and Spelling Condition for Type 1 Words

\begin{tabular}{|c|c|c|c|c|c|c|c|c|c|c|}
\hline \multirow[b]{3}{*}{ Age group/Spelling condition } & \multicolumn{10}{|c|}{ Rate of presentation (ms/word) } \\
\hline & \multicolumn{2}{|c|}{230} & \multicolumn{2}{|c|}{260} & \multicolumn{2}{|c|}{290} & \multicolumn{2}{|c|}{340} & \multicolumn{2}{|c|}{390} \\
\hline & $M$ & $S D$ & $M$ & $S D$ & $M$ & $S D$ & $M$ & $S D$ & $M$ & $S D$ \\
\hline \multicolumn{11}{|l|}{ Young adults } \\
\hline Correct spelling & .939 & .093 & .872 & .128 & .922 & .153 & & & & \\
\hline Unrepeated-letter misspellings & .278 & .193 & .329 & .249 & .307 & .221 & & & & \\
\hline Repeated-letter misspellings & .217 & .216 & .150 & .193 & .228 & .220 & & & & \\
\hline Repetition deficit ${ }^{a}$ & .061 & & .179 & & .079 & & & & & \\
\hline \multicolumn{11}{|l|}{ Older adults } \\
\hline Correct spelling & & & & & .833 & .182 & .872 & .152 & .872 & .167 \\
\hline Unrepeated-letter misspellings & & & & & .233 & .281 & .201 & .229 & .190 & .224 \\
\hline Repeated-letter misspellings & & & & & .111 & .188 & .200 & .270 & .206 & .260 \\
\hline Repetition deficit ${ }^{\mathrm{a}}$ & & & & & .122 & & .001 & & -.016 & \\
\hline
\end{tabular}

${ }^{a}$ Repetition deficits are the unrepeated condition minus the repeated condition.

The more complex, conditional measures described next were necessary to conclusively establish age-linked asymmetries in our retrieval data. The conditional measures in Figure 2 collapsed across rate and excluded retrieval trials on which participants failed to correctly perceive the stimuli in the detection task, thereby ensuring that detection effects did not contaminate subsequent retrieval effects, an essential precaution for establishing asymmetries between detection and retrieval. Conditional retrieval probability for correctly spelled stimuli is the probability of correct retrieval given correct perception that the stimulus was correctly spelled in the detection task and is shown as a function of age group in Figure 2 (right panel). Conditional retrieval probability

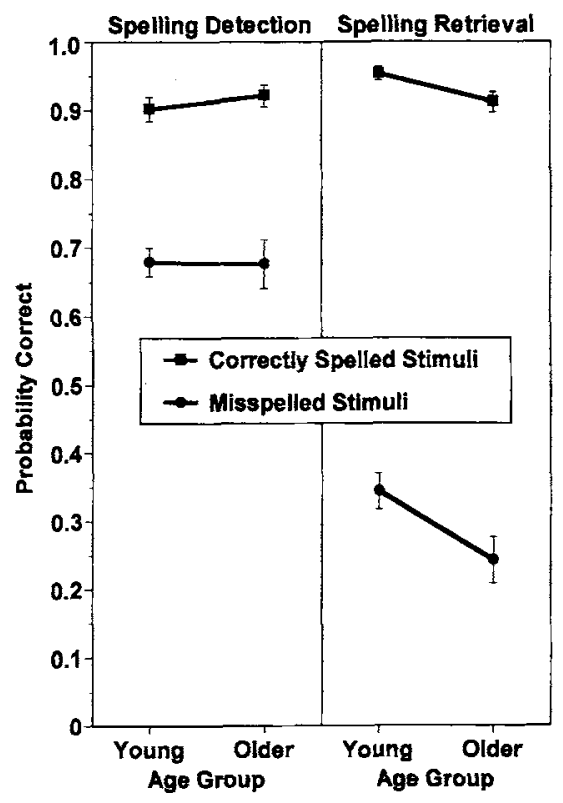

Figure 2. Probability of correct detection (left panel) for young and older adults and conditional probability of correct retrieval given correct detection (right panel) for correctly spelled and misspelled stimuli. for misspelled stimuli is the probability of correct retrieval given correct detection that the stimulus was misspelled in the detection task and is shown as a function of age group in Figure 2 (right panei). To simplify exposition, our results include only statistical analyses for these conditional retrieval measures, which were more appropriate and, in any case, led to the same basic conclusions as analyses of the unconditional data in Tables 3 and 4.

\section{The Asymmetry for Correctly Spelled Stimuli}

For correctly spelled Type 1 stimuli, a 2 (age) $\times 2$ (detection vs. retrieval task) multivariate analysis of variance (MANOVA) on detection probabilities and conditional retrieval probabilities in Figure 2 indicated no main effect of age $(F<1)$ or task, $F(1,70)$ $=2.70, M S E=0.01, p>.104$. However, the Age $\times$ Task interaction was significant, $F(1,70)=5.73, M S E=0.01, p<$ .019 , and further analyses indicated that detection probabilities did not differ for young and older adults $(F<1)$ but conditional retrieval probabilities were greater for young than older adults, $F(1,70)=5.30, M S E=0.01, p<.024$. This correct spelling asymmetry was not attributable to ceiling effects because performance was closer to ceiling for older adults in the detection task but closer to ceiling for young adults in the retrieval task (see Figure 2), so that ceiling effects would have worked against this asymmetry.

Correctly spelled Type 2 stimuli: A replication. An analysis of correctly spelled Type 2 words $(n=35)$ replicated the correct spelling asymmetry for a different set of stimuli and procedures. Table 5 shows the mean detection probabilities for correctly spelled Type 2 words as a function of age group, together with conditional retrieval probabilities given correct detection. A 2 (age) $\times 2$ (task: detection vs. conditional retrieval) MANOVA on these data indicated no main effect of age, $F(1,70)=1.42$, $M S E=0.01, p>.236$, or task, $F(1,70)=2.81, M S E=0.01, p>$ .097 , but there was a significant Age $\times$ Task interaction, $F(1,70)$ $=6.11, M S E=0.01, p<.016$. Further analyses indicated no age difference in detection, but there was a large age difference favoring the young participants in retrieval when correctly spelled 
Table 5

Detection Probabilities and Conditional Retrieval Probabilities for

All Type 2 Words and Type 2 Replacement Misspellings

\begin{tabular}{|c|c|c|c|c|c|c|c|c|}
\hline \multirow[b]{3}{*}{ Stimuli/Age group } & \multicolumn{4}{|c|}{ Detection probabilities } & \multicolumn{4}{|c|}{ Conditional retrieval probabilities } \\
\hline & \multicolumn{2}{|c|}{$\begin{array}{c}\text { All Type } 2 \\
\text { words }\end{array}$} & \multicolumn{2}{|c|}{$\begin{array}{c}\text { Type } 2 \\
\text { replacement } \\
\text { misspellings }\end{array}$} & \multicolumn{2}{|c|}{$\begin{array}{c}\text { All Type } 2 \\
\text { words }\end{array}$} & \multicolumn{2}{|c|}{$\begin{array}{c}\text { Type } 2 \\
\text { replacement } \\
\text { misspellings }\end{array}$} \\
\hline & $M$ & $S D$ & $M$ & $S D$ & $M$ & $S D$ & $M$ & $S D$ \\
\hline \multicolumn{9}{|l|}{ Correctly spelled } \\
\hline Young adults & .908 & .062 & & & .918 & .071 & & \\
\hline Older adults & .922 & .060 & & & .869 & .119 & & \\
\hline \multicolumn{9}{|l|}{ Misspelled } \\
\hline Young adults & .712 & .127 & .747 & .140 & .882 & .087 & .776 & .680 \\
\hline Older adults & .735 & .155 & .870 & .104 & .766 & .185 & .740 & .213 \\
\hline
\end{tabular}

Type 2 words were recognized as correctly spelled, $F(1,70)$ $=4.44, M S E=0.01, p<.039$. This correct spelling asymmetry for Type 2 words was not attributable to ceiling effects because older adults were closer to ceiling than young adults in the detection task but further from ceiling in the retrieval task (see Table 5).

Correlations with activities and background characteristics. Young and older adults differed at the .05 level on only one reported activity: Older adults reported spending significantly more hours per week doing crossword puzzles than young adults, $t(64)=2.31, p<, 024$. However, our main dependent measures (e.g., correct detection and written spelling retrieval for correctly spelled words) did not correlate reliably with time spent doing crossword puzzles at the .05 level for either young or older adults.

Multivariate analyses of covariance with education level and Nelson-Denny scores as covariates yielded only one reliable regression with our dependent measures for Type 1 words: Education correlated negatively with correct detection probability for older adults, $r(36)=-.385, p<.02$, an outcome that could only have worked against the correct spelling asymmetry because older adults had more education than young adults. This same correlation for young adults was positive, $r(36)=.312$, and unreliable at the .05 level.

\section{The Asymmetry for Misspelled Stimuli}

For misspelled Type 1 stimuli, Figure 2 (left panel) shows mean detection probabilities as a function of age, and Figure 2 (right panel) shows mean retrieval probabilities conditional on correct detection. A 2 (age) $\times 2$ (detection vs. retrieval task) MANOVA on these data yielded no main effect of age, $F(1,70)=1.98$, $M S E=0.05, p>.164$, but a main effect of task, $F(1,70)=$ 382.05, MSE $=0.01, p<.001$, with higher detection probabilities than retrieval probabilities. Consistent with a misspelling asymmetry, the Age $\times$ Task interaction was also significant, $F(1,70)$ $=6.09, M S E=0.01, p<.016$, with no age difference for the detection of misspellings $(F<1)$ but a large age difference favoring young adults for retrieval of misspellings that were detected as misspelled, $F(1,70)=5.53, M S E=0.03, p<.021$.

Misspelled Type 2 words: A replication. Analyses of misspelled Type 2 words $(n=20)$ replicated the misspelling asymmetry for a different set of stimuli and procedures. Table 5 shows the mean detection probabilities for Type 2 misspellings as a function of age group, together with conditional retrieval probabilities given correct detection. A 2 (age) $\times 2$ (task: detection vs. conditional retrieval) MANOVA on these data indicated no main effect of age, $F(1,70)=2.83, M S E=0.03, p>.096$, but a main effect of task, $F(1,70)=29.28, M S E=0.01, p<.001$, with better detection than retrieval. The Age $\times$ Task interaction was also significant, $F(1,70)=13.98, M S E=0.01, p<.001$, due to a misspelling asymmetry: There was no age difference in detection $(F<1)$ but a large age difference favoring young adults in conditional retrieval, $F(1,70)=11.75, M S E=0.02, p<.001$. This misspelling asymmetry for Type 2 words was not due to floor or ceiling effects because performance was closer to ceiling for older adults in the detection task but closer to ceiling for young adults in the retrieval task (see Table 5), indicating that ceiling effects would have worked against a misspelling asymmetry for Type 2 words. This replication indicates that the misspelling asymmetry cannot be attributed to stimulus and procedural factors specific to Type 1 misspellings.

Length-detection analyses. Type 2 replacement misspellings (e.g., quadrant misspelled as quadrent) exhibited the Age $\times$ Task (misspelling asymmetry) interaction at the .001 level (see Table 5) and were detected with higher probability than different-length (i.e., all other) misspellings ( $p<.001$ ). These findings rule out an "expected length account" in which detecting the unusual length of Type 1 misspellings provided the basis for the misspelling asymmetry. Although Type 1 stimuli always differed in length from the original or expected word, replacement misspellings were always the same length as the original word and could not have been detected on the basis of expected word length.

\section{Subsidiary Results Involving Rate and Repetition}

\section{Detection and Retrieval Analyses as a Function of Rate}

Rate had no effect on the probability of detecting either correctly or incorrectly spelled Type 1 stimuli. A 2 (age) $\times 2$ (correct vs. incorrect spelling) $\times 3$ (rate: fast, medium, and slow) MANOVA yielded a main effect of spelling condition, $F(1,70)=$ 123.16, MSE $=0.05, p<.001$, with better detection of correct than incorrect spelling, and a main effect of rate, $F(2,140)=3.15$, 
$M S E=0.01, p<.046$, with better detection at the slowest rate. However, there was no main effect of age and no reliable interactions involving rate (largest $F=2.98$, smallest $p=.054$ ). Similar analyses for detecting correctly and incorrectly spelled Type 2 words likewise yielded no effects of age or rate and no interactions involving rate. MANOVAs using only the data for the single rate shared by young and older adults ( $290 \mathrm{~ms} /$ word) likewise yielded no age effects or interactions involving age. Independent MANOVAs for retrieval of correctly and incorrectly spelled words that were detected as such also yielded no effects of rate and no Age $\times$ Rate interactions for either Type 1 or Type 2 stimuli. These findings indicate that rate was not a factor on either the detection side or the production side of the misspelling and correct spelling asymmetries.

\section{Symmetric Effects of Repetition for Task and Age}

The double dissociation data. To determine whether repetition would exhibit the same interaction with aging or with task as the misspelling asymmetry, we conditionalized correct recall of repeated- and unrepeated-letter misspellings on correct detection as before, thereby ensuring that effects of repetition on perception did not influence or contaminate effects of repetition on retrieval. ${ }^{3}$ We also took two steps to ensure that effects of repetition on retrieval were independent of age-linked declines in the ability to spell (see Figure 2; also MacKay \& Abrams, 1998). One was to define correct retrieval as correct inclusion of the added or target letter (i.e, correct spelling of the entire word was unnecessary). The other was to conditionalize correct target recall on inclusion of the pretarget letter: Responses containing missing pretargets (e.g., wildierness misproduced as weldness, and calendear misproduced as calander) were discarded for logical and theoretical reasons that have been noted in many recent studies of RD (see, e.g., MacKay et al., 1994; MacKay, Abrams, Pedroza, \& Miller, 1996). If a participant does not perceive the pretarget, a repeated-target trial is unrepeated from a psychological point of view, so that including these trials in the means would underestimate the true degree of retrieval RD. These "double conditional analyses" met the criterion of equivalent rates of data discard across groups (see MacKay et al., 1994; MacKay \& Miller, 1996): 31\% for young adults and $27 \%$ for older adults, with only 2 participants lost because of empty cells in our main MANOVAs.

Figure 3 shows mean detection probabilities (left panel) and double conditional probabilities of correct retrieval (right panel) for repeated- and unrepeated-letter targets as a function of age group collapsed across the three rates. A 2 (age) $\times 2$ (repetition) $\times 2$ (task: detection vs. retrieval) MANOVA on these data revealed a main effect of age, $F(1,68)=4.44, M S E=0.07, p<$ .039 , with better performance for the young than older adults; a main effect of repetition, $F(1,68)=244.10, M S E=0.04, p<$ .001 , with better performance for unrepeated- than repeated-letter targets; and a main effect of task, $F(1,68)=12.80, M S E=0.05$, $p<.001$, with better performance in the detection task than in the retrieval task. The Age $\times$ Task interaction was also significant, $F(1,68)=9.98, M S E=0.05, p<.002$, with no age difference in detection $(F<1)$ but a large age difference favoring the young in target recall, $F(1,68)=12.05, M S E=0.07, p<.001$. No other interactions were significant (smallest $p>.154$ ). Similar analyses using only the single rate shared by young and older adults gave

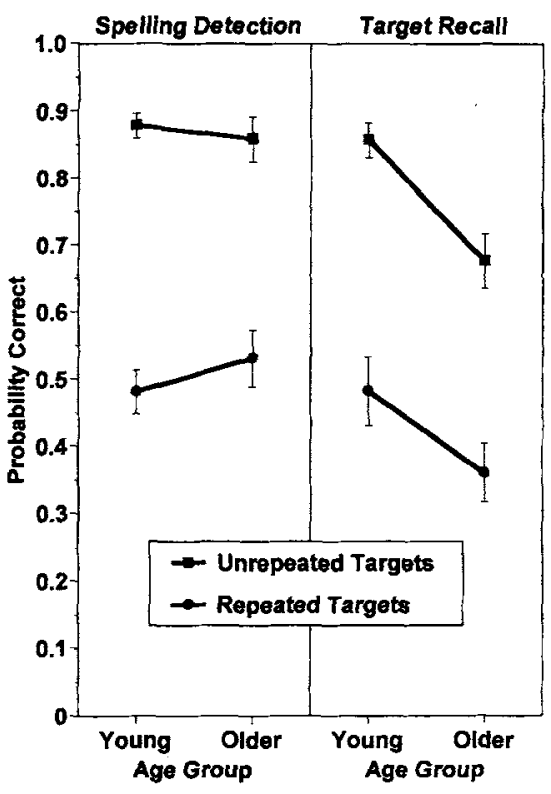

Figure 3. Probability of correct detection (left panel) for young and older adults and double conditional probability of correct target-letter retrieval (given correct detection and pretarget recall) for repeated- and unrepeatedletter misspellings (right panel).

identical results. These results indicate that repetition has powerful effects that are symmetrical with respect to task (i.e., the effects of repetition were similar in detection and recall) and with respect to age (i.e., the effects of repetition were similar for young and older adults).

Floor and ceiling effect analyses. Because detection probabilities for repeated-letter misspellings approximated coin toss levels of accuracy (.5) for both the young and older adults (see Figure 3, left panel), our participants may have been simply guessing in this condition, effectively introducing a $50 \%$ floor that might have eliminated a potential Age $\times$ Repetition interaction in the detection data. We therefore divided older participants into two groups with detection levels for repeated-target misspellings that were above versus below their group median; we did the same for young participants. Performance for both the young and older above-therepeated-median groups was well above the hypothesized $50 \%$ floor, but a comparison of these groups yielded no Age $\times$ Repetition interaction $(F<1)$, ruling out this "guessing floor" account of the misspelling asymmetry. Three additional MANOVAs on data involving split groups ruled out other floor and ceiling accounts of the nonsignificant Age $\times$ Repetition interactions.

\footnotetext{
${ }^{3}$ We conducted single conditional analyses that ignored this condition, with results that indicated dramatic age-linked floor effects. If Figure 3 had presented single conditional probabilities of correct retrieval (i.e., given only correct pretarget recall), these "hidden floor effects" would have led to the erroneous conclusion that young adults exhibit a greater retrieval repetition deficit than older adults (see the Appendix for theoretical implications of this error).
} 


\section{Discussion}

\section{Accounts of Asymmetries Involving Correctly Spelled Stimuli}

What accounts for the correct spelling asymmetry? Why was there a large age-linked deficit in retrieving the correct spelling of words that were recognized as correctly spelled, but no age-linked deficit in detecting that the same words were correctly spelled? The correct spelling asymmetry is consistent with the asymmetry hypothesis, that perception and production are not mirror-image processes and that aging has disproportionately greater effects on production than perception. One particular asymmetry hypothesis, the TDH of MacKay and Burke (1990), explains the correct spelling asymmetry and a wide range of other findings suggesting age-linked asymmetries between production and perception in terms of the differing structure of top-down versus bottom-up connections. Age effects are greatest under the TDH when a node critical to a task receives priming from only a single source or connection within the network, and this "single source condition" holds for the top-down connections for retrieving both phonology and orthography (see Figure 1). Age-linked deficits on the output side in the present study are therefore consistent with the TDH and with the many studies reviewed elsewhere (e.g., MacKay \& Abrams, 1998) that demonstrate age-linked deficits in retrieving the phonology and orthography of familiar words.

Turning to the input side, age effects are small or nonexistent under the TDH when a node critical to a task receives priming simultaneously from more than one source or connection within the network, and this "multiple source condition" holds for the bottom-up connections for perceiving familiar words within the phonological and orthographic system (see Figure 1). The age constancy in perceiving correctly spelled words as correctly spelled in the present experiment is therefore consistent with the TDH and with the many studies noted in the introduction that demonstrate small or nonexistent age effects on the input side.

We turn now to some unlikely accounts of the correct spelling asymmetry. One is the hypothesis that cohort-related educational deficiencies affected the ability of older adults to retrieve the spelling of high-frequency words in the present study: Contrary to this hypothesis, the older adults were more educated than the young adults, and they rated the rigor with which their grade schools taught spelling skills no less highly than young adults. Moreover, the education level of older adults correlated negatively with the ability to detect whether words were correctly or incorrectly spelled, an outcome that could only have worked against the correct spelling asymmetry. Our results also rule out other possible cohort effects related to how many hours per week participants spent reading, writing, and solving crossword puzzles: Only one of these factors correlated reliably with cohort, and it was unrelated to the probability of detecting and retrieving correct spelling.

The form of the correct spelling asymmetry also rules out sensory- or receptor-level artifacts in the asymmetrical effects of aging on the input versus output side: Age-linked sensory deficits could cause only increased perceptual errors for older relative to young adults for correctly spelled words, contrary to the present results. Furthermore, the correct spelling asymmetry cannot be explained in terms of task difficulty such that older adults perceived correctly spelled words as correctly spelled in the perception task but recalled these words as misspelled in the production task because the perception task was easier or "less complex" than the production task and therefore less susceptible to age-linked declines in performance. Contrary to this hypothesis, the performance level of young adults was numerically higher for the production task than for the perception task (see Figure 2), which suggests that, independent of the age factor, the perception task was at least as difficult as the production task in the case of correctly spelled stimuli. Performance for correctly spelled words was also numerically higher for older adults in the production task than for young adults in the perception task (see Figure 2), suggesting that the production task per se was not difficult for the older adults. The similar pattern of results for Type 2 words (see Table 5) also argues against a task difficulty hypothesis.

Another unlikely account of the correct spelling asymmetry points to the fact that participants always executed the perception task before the production task. Consequently, older adults may have forgotten perceived details of the stimuli at the time of recall and therefore tended to misrecall correctly spelled words as misspelled more often than young adults. Contrary to this hypothesis, however, performing the yes-no response in the detection task took about $1 \mathrm{~s}$, and young and older adults usually do not differ on memory tasks with delays of that magnitude (see Craik, 1977, for a review). There is also no reason to believe that when recalling a correctly spelled word that they perceive to be correctly spelled, older adults cannot remember a single item of information ("correct") for a second or two. In addition, if forgetting was the basis for the correct spelling asymmetry, then contrary to present data, age differences should have been smaller in retrieving frequently encountered, correctly spelled words than in retrieving misspelled forms that they had encountered only once. Moreover, having the perception task precede the production task provides the strongest possible case for a correct spelling asymmetry. If the production task had preceded the perception task, errors on the perception task could be attributed both to forgetting and to attempts to maintain consistency with the prior written response, which would more likely be in error for older than young adults (see MacKay \& Abrams, 1998). Or given a recall task with no prior perception task, it could be argued that older adults more often misperceived the stimulus or forgot whether it was correctly or incorrectly spelled while writing down their response. As it was, we observed an age-linked increase in errors on the production task even when participants just saw the correctly spelled word and even though they probably tried to maintain consistency with their (correct) response in the detection task. Furthermore, this age-linked increase in retrieval errors occurred even though the older adults could see what they had written and determine whether, when read back, it matched what they meant to write.

Another unlikely account of the correct spelling asymmetry is that our observed age-linked declines in spelling retrieval reflect transient or readily correctable errors. MacKay and Abrams (1998) ruled out this hypothesis using procedures that deemphasized response speed and minimized the likelihood of transient errors (e.g., self-paced trials, slowly presented stimuli). Consistency of 
our results with those of MacKay and Abrams therefore suggests that misspellings of correctly spelled words by older adults in the present study were not transient errors in spelling retrieval. ${ }^{4}$ MacKay and Abrams also ruled out other factors that might seem relevant to the present age-linked deficits in retrieving correct spelling: a variety of potential cohort effects, general slowing in older adults, and declines in the ability of older adults to monitor or detect errors in their written responses. At the same time, however, our results go beyond MacKay and Abrams's findings by indicating that older adults will often misspell a word despite having just seen the word spelled correctly and despite having just responded that the word was spelled correctly.

Another unlikely account of the correct spelling asymmetry is the hypothesis that the perception of familiar, correctly spelled words involves wholistic or gestaltlike processes that are rapid, automatic, and parallel in nature (see Barron, 1980) and therefore immune to the effects of aging, whereas retrieval of correct spellings is slow, deliberate, and sequential in nature and therefore susceptible to an age-linked decline. This hypothesis fails to explain the parallel nature of the correct spelling and misspelling asymmetries in our data and predicts that replacement misspellings (e.g., quadrant misspelled as quadrent), which resembled the original, correctly spelled word in overall shape or wholistic Gestalt more so than misspellings formed by adding a letter (e.g., endeavor misspelled as endeavuor) or by omitting a letter (e.g., handkerchief misspelled as hankerchief), should not exhibit a misspelling asymmetry and should give rise to more misperceptions than the addition or omission misspellings, contrary to the present results.

Another unlikely account of the correct spelling asymmetry is the symmetry hypothesis outlined in the introduction: Theories that attribute cognitive aging to a single factor, such as general slowing, and view perception and production as mirror-image processes do not predict the correct spelling asymmetry and can explain it only as an artifact. However, the use of exactly the same participants and stimuli in our perception and production tasks rules out two possible artifacts in the many studies that have examined the effects of aging separately on perception and production. Results discussed earlier also rule out artifacts related to cohort, age-linked sensory deficits, ceiling and floor effects, task difficulty effects, task-order effects, insensitivity of our perceptual measure due to chance-level responding, and insufficient power in our design (see the Appendix).

\section{Accounts of Asymmetries Involving Misspelled Stimuli}

We observed no age-linked deficits in detecting that a word was misspelled, but we did find large age-linked deficits in retrieving the spelling of a word that participants recognized as misspelled. This misspelling asymmetry is consistent with predictions of the asymmetry hypothesis discussed earlier and is embodied in theories such as NST, in which perception and production are not mirror-image processes and in which cognitive aging impairs production more so than perception.

Results for misspelled words did not support the symmetry hypothesis embodied in theories that attribute cognitive aging to a single factor such as general slowing and view perception and production as mirror-image processes. Under the symmetry hypothesis, the misspelling asymmetry is an artifact, but our results did not support artifactual accounts of the misspelling asymmetry based on age-linked sensory deficits, ceiling and floor effects, task-order effects, different activities or background characteristics of our participants, different stimuli in the perception and production tasks, insensitivity of our perceptual measure due to chancelevel responding, and insufficient power in our design (see the Appendix). The misspelling asymmetry was also not due to lengthdetection processes involving Type 1 misspellings: Analyses of Type 2 replacement misspellings (which did not differ in length from the original word) ruled out this hypothesis. Furthermore, the misspelling asymmetry was not due to task difficulty because the performance level of the young adults was greater for retrieving than for detecting Type 2 misspellings (see Table 5), suggesting that, independent of the age factor, the perception task was at least as difficult as the production task for misspelled words.

Also problematic for symmetry theories, at least one factor that affects the retrieval of misspellings does not exhibit the same interaction with either age or task as the misspelling asymmetry. Specifically, repetition had strong effects that were symmetrical or equivalent in magnitude for both young and older adults and for both the detection and retrieval tasks. This double dissociation pattern suggests that the misspelling asymmetry is age-specific and must be explained by theories of cognitive aging.

\section{Subsidiary Issues}

\section{Aging and Error Detection}

The ability to detect whether a correctly spelled word was correctly spelled remained constant with age in the present data, and so did the ability to detect whether misspelled words were misspelled, consistent with the asymmetry hypothesis, and with the results of Mahoney (1997), that young and older adults are equally able to detect self-produced phonological errors. Nonetheless, our results are inconsistent with the report of Valencia-Laver (1992) that older adults correct (and presumably detect) selfproduced lexical substitution errors (e.g., "Turn left, I mean, right") reliably less often than young adults in a route description task (see also MacKay, 1992). Perhaps age constancy in error detection holds only for some types of errors or some levels of language.

Our error detection results are also relevant to the processing resolution hypothesis of Allen, Madden, and Slane (1995), Allen, Madden, Weber, and Groth (1993), and Stadtlander (1995) that perceiving letter-level codes demands high processing resolution that is especially difficult for older adults because age-linked

\footnotetext{
${ }^{4}$ A more complex but equally implausible transient error hypothesis can be constructed from two sources. One is the demonstration of Jacoby and Hollingshead (1990) and others that correct spelling exhibits a transient decline for a brief period after reading similar but misspelled words, and the other is the hypothesis of Balota and Ferraro (1993) that general slowing, inhibitory deficits, and age-linked interactions between speed and accuracy may combine in complex ways to cause age-linked but transient errors in speeded response tasks. MacKay and Abrams's (1998) data contradict this more complex transient error hypothesis, as do our analyses of the rate factor (see the Appendix). Moreover, an inhibition deficit hypothesis would predict neither the present Age $\times$ Task interactions nor the comparable age differences in retrieving correctly and incorrectly spelled words (see Figure 2).
} 
"internal noise randomly changes visual features" at a central perceptual level (i.e., independent of age-linked sensory deficits). However, if aging causes random changes in visual features during perception, the ability to detect correct spelling should decline with aging in the present paradigm because a correctly spelled word with altered visual features should often be misperceived as misspelled. The fact that detecting correct spelling remained constant with aging in the present data therefore contradicts the processing resolution hypothesis. Similarly, older adults with deficits in processing resolution should be less able to detect added-letter misspellings than young adults, contrary to the present data indicating age constancy. One possible resolution of these discrepant conclusions is that the Allen et al. task involved perception of words typed in novel formats (mixed upper- and lowercase, together with unusual patterns of letter spacing) that may have required new connection formation. The Allen et al. results may therefore reflect well-established age deficits in new connection formation (see, e.g., MacKay \& Burke, 1990, for a review) rather than age-linked declines in processing resolution.

Our observed age constancy in detecting misspellings also contradicts the hypothesis that, because of sensory deficits, greater experience with language, or both, older adults do not process each letter in familiar words but tend to rely on "context," in this case, the phonological and orthographic redundancy that exists within correctly spelled words. Such "reliance on context" might enable older adults to detect that correctly spelled words are correctly spelled without processing each letter, but should frequently cause older adults to misperceive misspelled words as correctly spelled. This is inconsistent with our finding that detecting misspellings remained constant as a function of age.

Our observed age constancy in detecting repeated- and unrepeated-letter misspellings also contradicts another seemingly plausible hypothesis: that the ability to detect novelty is especially susceptible to effects of aging and that novelty plays a role in detecting unrepeated-letter misspellings such as elderkly because the added letter is novel to the word but plays no role in detecting repeated-letter misspellings such as elderdly because the added letter is a familiar component of the word. This novelty detection hypothesis predicts a much larger age-linked deficit in detecting unrepeated-letter misspellings than in detecting repeated-letter misspellings, contrary to data.

\section{Effects of Aging on RD}

The effects of aging on $\mathrm{RD}$ in the present paradigm are relevant to the issue of whether there is more than one type of RD. RD in the present paradigm was similar in magnitude for perception and retrieval, similar in magnitude across a wide range of presentation rates, and similar in magnitude for young and older adults. This pattern contrasts with results for standard-paradigm $\mathrm{RD}$, in which RD has been shown to decrease as a linear function of presentation time and in which older adults exhibit more RD than young adults across a wide range of presentation rates (MacKay et al., 1994). Perhaps these contrasting empirical results reflect methodological differences between the standard RSVP paradigm and the present paradigm (see the Appendix for detailed procedural comparisons).

On the other hand, perhaps the contrasting results have a parsimonious explanation in terms of some single underlying cause. We do not currently understand how this "universal RD mecha- nism" might work. However, to stimulate research that may help explain it, we tentatively postulate two theoretically distinct classes of RD: surface versus deep RD, analogous to the concepts of surface versus deep dyslexia and surface versus deep dysgraphia (see, e.g., Parkin, 1996, pp. 167-180). Surface RD occurs in both detection and retrieval, reflects the repeated activation within a brief period of existing nodes representing highly practiced orthographic and phonological units in familiar words, and does not interact with aging (see the Appendix). In contrast, deep RD occurs only in sentence and phrase processing, it increases as a function of aging, and it is linked to the process that connects words to phrases (see MacKay \& Miller, 1996; MacKay et al., 1994). Whether deep RD occurs in both perception and retrieval has yet to be established for sentences in the standard RSVP paradigm. However, deep RD is strongly influenced by syntactic and semantic factors and underlies the semantic blindness phenomenon demonstrated by MacKay and Miller (1994). MacKay and Abrams (1994) also demonstrated a type of deep RD in lists that increases in magnitude when repeated words occur in familiar phrases such as good night and night gown rather than in lists of unrelated words. Because phrases are fundamentally syntactic and semantic entities, this finding is consistent with the semantic blindness phenomenon and with a role for syntactic and semantic factors in the RSVP paradigm.

Results of Abrams, Dyer, and MacKay (1996) with a modified RSVP procedure also support the existence of deep RD: In their procedure, each RSVP frame contained either a complete phrase, as in [They wanted] [to play sports] [but sports] [were not allowed], or a nonphrase, as in [They wanted to] [play sports but] [sports were not] [allowed]. Equated on average across the phrasecongruent and phrase-incongruent sentences were the actual words, time per word, frames per sentence, mean words per frame, serial position of pretarget and target words within the frames, and the absolute and relative "eccentricity" of target and pretarget words (i.e., their degree of shift to the left or right of central fixation). The results indicated a significant increase in RD for phrase-incongruent relative to phrase-congruent RSVP sentences, consistent with an effect of syntactic and semantic factors, such that deep RD decreases or increases in magnitude depending on whether the RSVP procedure makes it easier or more difficult to form word-to-phrase links in sentences. Miller and MacKay (1996) reported analogous effects for repetition deafness in sentences that are likewise consistent with the existence of semantic blindness and with the effects of semantic and syntactic factors on deep RD, and MacKay and Miller (1996) completed the picture by showing that aging has identical (exacerbating) effects on repetition deafness and blindness in sentences.

\section{Conclusion}

The results of this study demonstrate age-linked asymmetries predicted under NST between detecting versus retrieving orthographic information. When young and older adults saw briefly presented, high-frequency words that were either correctly or incorrectly spelled, their performance on a perceptual task (signaling whether the word was misspelled) exhibited two age-linked asymmetries relative to performance on a production task (i.e., writing out what they had seen). The correct spelling asymmetry was that older adults perceived correctly spelled words as correctly 
spelled as readily as young adults but were less able to immediately retrieve the correct spelling than young adults. The misspelling asymmetry was that older adults detected that a word was misspelled as readily as young adults but were less able to immediately retrieve the misspelling than young adults. Neither asymmetry was attributable to artifacts such as task complexity, floor or ceiling effects, general slowing, stimulus-specific characteristics, sensory-level factors, cohort-related activities, or background characteristics of our participants. In addition, our double dissociation procedures indicated that the misspelling asymmetry was specific to aging and required explanation within theories of cognitive aging. Both asymmetries also direct a more general challenge to theories of cognitive aging, namely to explain why some aspects of information processing are more vulnerable to the effects of aging than others: Theories that predict either sparing or impairment of verbal abilities across the board in old age are inconsistent with these asymmetries. However, both asymmetries are consistent with NST and with similar age-linked asymmetries that can be discerned in a wide range of other studies that have examined effects of aging independently on the input and output side of language.

\section{References}

Abrams, L., Dyer, J. R., \& MacKay, D. G. (1996). Repetition blindness interacts with syntactic grouping in rapidly presented sentences. Psychological Science, 7, 100-104.

Allen, P. A., Madden, D. J., \& Slane, S. (1995). Visual word encoding and the effect of adult age and word frequency. In P. A. Allen \& T. R. Bashore (Eds.), Age differences in word and language processing (pp. 30-71). Amsterdam: North-Holland.

Allen, P. A., Madden, D. J., Weber, T. A., \& Groth, K. E. (1993). Influence of age and processing stage on visual word recognition. Psychology and Aging, 8, 274-282.

Au, R., Joung, P., Nicholas, M., Obler, L. K., Kass, R., \& Albert, M. L. (1995). Naming ability across the adult life span. Aging and Cognition, 2, 300-311.

Balota, D. A., \& Ferraro, F. R. (1993). A dissociation of frequency and regularity effects in pronunciation performance across young adults, older adults, and individuals with senile dementia of the Alzheimer type. Journal of Memory and Language, 32, 573-592.

Barron, R. W. (1980). Visual and phonological strategies in reading and spelling. In U. Frith (Ed.), Cognitive processes in spelling (pp. 195213). New York: Academic Press.

Birren, J. E., \& Woodruff, D. S. (1983). Aging: Past and future. In D. S. Woodruff \& J. E. Birren (Eds.), Aging: Scientific perspectives and social issues (2nd ed., pp. 115-116). Monterey, CA: Brooks/Cole.

Bowles, N. L., \& Poon, L. W. (1985). Aging and retrieval of words in semantic memory. Journal of Gerontology, 40, 71-77.

Burke, D. M. (1997). Language, aging, and inhibitory deficits: Evaluation of a theory. Journal of Gerontology: Psychological Sciences, 52B, $\mathrm{P} 254-\mathrm{P} 264$.

Burke, D. M., \& MacKay, D. G. (1997). Memory, language, and aging. Philosophical Transactions of the Royal Society, Biological Sciences, 352, 1845-1856.

Burke, D. M., MacKay, D. G., Worthley, J. S., \& Wade, E. (1991). On the tip of the tongue: What causes word finding failures in young and older adults? Journal of Memory and Language, 30, 542-579.

Burke, D. M., \& Yee, P. L. (1984). Semantic priming during sentence processing by young and older adults. Developmental Psychology, 20, 903-910.

Cohen, G., \& Faulkner, D. (1983). Word recognition: Age differences in contextual facilitation effects. British Journal of Psychology, 74, 239251 .

Cohen, J. D., MacWhinney, B., Flatt, M., \& Provost, J. (1993). PsyScope: A new graphic interactive environment for designing psychology experiments. Behavioral Research Methods, Instruments and Computers, 25, 257-271.

Connelly, S. L., Hasher, L., \& Zacks, R. T. (1991). Age and reading: The impact of distraction. Psychology and Aging, 6, 533-541.

Craik, F. I. M. (1977). Age differences in human memory. In J. E. Birren \& K. W. Schaie (Eds.), Handbook of the psychology of aging (1st ed., pp. 384-420). New York: Van Nostrand Reinhold.

Fagot, C., \& Pashler, H. (1995). Repetition blindness: Perception or memory failure? Journal of Experimental Psychology: Human Perception and Performance, 21, 275-292.

Faucett, L., \& Maki, I. (1932). A study of English word values. Tokyo: Matsumura Sanshodo.

Francis, W. N., \& Kucera, H. (1982). Frequency analysis of English usage: Lexicon and grammar. Boston: Houghton Mifflin.

Hasher, L., \& Zacks, R. T. (1988). Working memory, comprehension, and aging: A review and a new view. In G. H. Bower (Ed.), The psychology of learning and motivation (pp. 193-225). New York: Academic Press.

Hochhaus, L., \& Johnston, J. C. (1996). Perceptual repetition blindness effects. Journal of Experimental Psychology: Human Perception and Performance, 22, 355-356.

Hochhaus, L., \& Marohn, K. M. (1991). Repetition blindness depends on perceptual capture and token individuation failure. Journal of Experimental Psychology: Human Perception and Performance, 17, 422-432.

Humphreys, G. W., Besner, D., \& Quinlan, P. T. (1988). Event perception and the word repetition effect. Journal of Experimental Psychology: General, 117, 51-67.

Jacoby, L. L., \& Hollingshead, A. (1990). Reading student essays may be hazardous to your spelling: Effects of reading correctly and incorrectly spelled words. Canadian Joumal of Psychology, 44, 345-358.

Kanwisher, N. G., \& Potter, M. C. (1990). Repetition blindness: Levels of processing. Journal of Experimental Psychology: Human Perception and Performance, 16, 30-47.

Kline, D. W. (1984). Processing sense information. In J. Botwinick (Ed.), Aging and behavior: A comprehensive integration of research findings (pp. 207-228). New York: Springer.

Laver, G. D., \& Burke, D. M. (1993). Why do semantic priming effects increase in old age? A meta-analysis. Psychology and Aging, 8, 34-43.

Levin, J. (1997). Overcoming feelings of powerlessness in "aging" researchers: A primer on statistical power in analysis of variance designs. Psychology and Aging, 12, 84-106.

Light, L. L., Valencia-Laver, D., \& Zavis, D. (1991). Instantiation of general terms in young and older adults. Psychology and Aging, 6, 337-351.

MacKay, D. G. (1969). The repeated letter effect in the misspellings of dysgraphics and normals. Perception \& Psychophysics, 5, 102-106.

MacKay, D. G. (1987). The organization of perception and action: A theory for language and other cognitive skills. New York: SpringerVerlag.

MacKay, D. G. (1992). Awareness and error detection: New theories and research paradigms. Consciousness and Cognition, 1, 199-225.

MacKay, D. G., \& Abrams, L. (1994, April). Repetition blindness, chunking, and the connection specificity hypothesis. Poster presented at the Cognitive Aging Conference, Atlanta, GA.

MacKay, D. G., \& Abrams, L. (1996). Language, memory, and aging: Distributed deficits and the structure of new-versus-old connections. In J. E. Birren \& K. W. Schaie (Eds.), Handbook of the psychology of aging (4th ed., pp. 251-265). San Diego, CA: Academic Press.

MacKay, D. G., \& Abrams, L. (1998). Age-linked declines in retrieving orthographic knowledge: Empirical, practical, and theoretical implications. Psychology and Aging, 13, 647-662. 
MacKay, D. G., Abrams, L., Pedroza, M. J., \& Miller, M. D. (1996). Cross-language facilitation, semantic blindness, and the relation between language and memory: A reply to Altarriba and Soltano. Memory \& Cognition, 24, 712-718.

MacKay, D. G., \& Burke, D. M. (1990). Cognition and aging: A theory of new learning and the use of old connections. In T. M. Hess (Ed.), Aging and cognition: Knowledge organization and utilization (pp. 213-263). Amsterdam: North-Holland.

MacKay, D. G., \& Miller, M. D. (1994). Semantic blindness: Repeated concepts are difficult to encode and recall under time pressure. Psychological Science, 5, 52-55.

MacKay, D. G., \& Miller, M. D. (1996). Can cognitive aging contribute to fundamental psychological theory? Repetition deafness as a test case. Aging, Neuropsychology, and Cognition, 3, 169-186.

MacKay, D. G., Miller, M. D., \& Schuster, S. P. (1994). Repetition blindness and aging: Evidence for a binding deficit involving a single, theoretically-specified connection. Psychology and Aging, 9, 251-258.

Madden, D. J. (1988). Adult age differences in the effects of sentence context and stimulus degradation during visual word recognition. $P_{s y-}$ chology and Aging, 3, 167-172.

Mahoney, P. G. (1997). Language production, speech errors, and aging. Unpublished doctoral dissertation, Claremont Graduate University.

McDowd, J. M., Oseas-Kreger, D. M., \& Filion, D. L. (1995). Inhibitory processes in cognition and aging. In F. Demster (Ed.), New perspectives on interference and inhibition in cognition (pp. 363-400). New York: Academic Press.

Miller, M. D., \& MacKay, D. G. (1994). Repetition deafness: Repeated words in computer compressed speech are difficult to encode and recall. Psychological Science, 5, 47-51.

Miller, M. D., \& MacKay, D. G. (1996). Relations between language and memory: The case of repetition deafness. Psychological Science, 7 , 347-351.

Mitchell, D. B. (1989). How many memory systems? Evidence from aging. Journal of Experimental Psychology: Learning, Memory, and Cognition, 15, 31-49.

Norback, P. G. (1974). The misspeller's dictionary. New York: Quadrangle/The New York Times Book Co.

Parkin, A. J. (1996). Explorations in cognitive neuropsychology. Cambridge, MA: Blackwell.

Rastle, K. G., \& Burke, D. M. (1996). Priming the tip of the tongue: Effects of prior processing on word retrieval in young and older adults. Journal of Memory and Language, 35, 586-605.

Stadtlander, L. M. (1995). Age differences in orthographic and frequency neighborhoods. In P. A. Allen \& T. R. Bashore (Eds.), Age differences in word and language processing (pp. 72-86). Amsterdam: North-Holland.

Stine, E. A. L., \& Wingfield, A. (1994). Older adults can inhibit highprobability competitors in speech recognition. Aging and Cognition, 1, $152-157$.

Valencia-Laver, D. L. (1992). Adult age differences in the production, detection, and repair of speech errors. Unpublished doctoral dissertation, Claremont Graduate University.

Welford, A. T. (1977). Serial reaction times, continuity of task, singlechannel effects and age. In S. Dornic (Ed.), Attention and performance $V I$ (pp. 79-97). Hillsdale, NJ: Erlbaum.

Wingfield, A., Alexander, A. H., \& Cavigelli, S. (1994). Does memory constrain utilization of top-down information in spoken word recognition? Evidence from normal aging. Language and Speech, 37, 221-235.

Woodward, J. A., Bonett, D. G., \& Brecht, M. L. (1990). Introduction to linear models and experimental design. San Diego, CA: Harcourt Brace Jovanovich.

\section{Appendix}

\section{Theory-Based Hypotheses and Results Related to Aging and Repetition Deficits}

\section{The Inhibition Repetition Deficit Hypothesis}

Inhibition is a frequently postulated cause of repetition deficit (RD) in rapid serial visual presentation (RSVP) sentences, and MacKay (1969) specifically hypothesized a self-inhibition process as the cause of $\mathrm{RD}$ in detecting and retrieving repeated-letter misspellings: According to this hypothesis, an activated letter node becomes difficult to activate again for a brief period, so that under time pressure, the second instance of a letter becomes difficult to detect and, if detected, becomes difficult to retrieve during recall. Because older adults suffer from inhibition deficits or agelinked declines in the effectiveness of processes that in theory involve neural inhibition (see Birren \& Woodruff, 1983; Pavlov, as described in McDowd, Oseas-Kreger, \& Filion, 1995), ${ }^{\mathrm{A}}$ the inhibition RD hypothesis predicts that young adults should exhibit more RD than older adults. However, the present data did not support this inhibition hypothesis prediction: $\mathrm{RD}$ was no greater for young than older adults in detecting misspellings or in reproducing detected misspellings.

\section{The Binding RD Hypothesis}

RD in RSVP sentences has been attributed to a difficulty in rapidly forming the new connections for binding repeated words into phrases for sentence comprehension and recall (see, e.g., Miller \& MacKay, 1994, 1996). Because older adults normally require more time than young adults to form new connections, aging should therefore exacerbate the problem that causes $\mathrm{RD}$ under this binding $\mathrm{RD}$ hypothesis, and, consistent with this prediction, MacKay, Miller, and Schuster (1994) showed that older adults exhibit more RD than young adults in recalling RSVP sentences. If, as MacKay and Abrams (1994) suggested, the present paradigm is theoretically and empirically analogous to the standard $\mathrm{RD}$ paradigm involving RSVP words, then older adults should exhibit greater orthographic RD than young adults. By the same reasoning, older adults should also retrieve the target letter in the wrong sequential position in a word more often than young aduits, and more so for repeated- than unrepeated-letter misspellings because time pressure will make it even more difficult for older adults to bind a target, and especially a repeated target (e.g., the added $i$ in hospitiable) to its novel sequential position in a word.

However, the present data supported neither of these predictions. First, $\mathrm{RD}$ was no greater for older than young adults in detecting or in reproducing Type 1 misspellings, and additional analyses indicated that this finding was not attributable to ceiling, floor, or level-of-performance effects; to differences between double conditional, single conditional, and unconditional data; to age-unrelated background characteristics of the participants; or to processes specific to our tasks or stimuli (e.g., error detection based on the abnormal word length of added-letter misspellings). Second, when the target letter was recalled, its position was accurately reproduced no more often for unrepeated- than repeated-letter targets, with

A1 As Burke (1997) pointed out, it remains to be shown that age-linked declines in empirical interference effects related to irrelevant information (as in, e.g., Connelly, Hasher, \& Zacks, 1991; Hasher \& Zacks, 1988) in principle involve neural inhibition. 
a trend in the opposite direction, that is, fewer positional errors involving repeated- $(38 \%)$ than unrepeated-targets $(45 \%)$, for both young and older adults, and age did not interact with position recall, contrary to the general binding RD hypothesis of MacKay and Abrams (1994).

The contrast between the present results and those of MacKay et al. (1994) using the standard RSVP paradigm for demonstrating RD therefore calls for detailed procedural and theoretical comparisons between these studies. One procedural difference is that participants in the standard paradigm simply recall the RSVP words in the order presented, which makes it impossible to determine whether RD is occurring during perception, during retrieval, or both in this paradigm (see also Fagot \& Pashler, 1995; Hochhaus \& Johnston, 1996). The present paradigm clarified this issue by demonstrating that $\mathrm{RD}$ is both a perceptual phenomenon (because detection was better for unrepeated- than repeated-letter misspellings) and a retrieval phenomenon (because retrieval was better for unrepeated- than repeated-letter misspellings). However, neither perceptual RD nor retrieval RD interacted with aging in the present data, ruling out the perception versus retrieval dimension as a viable basis for explaining the differing age effects in MacKay et al. (1994) versus the present study.

Another distinguishing procedural feature is that the present paradigm presents the repeated elements simultaneously ${ }^{\mathrm{A} 2}$ in different spatial positions, whereas the standard RSVP paradigm presents the repeated elements sequentially in the same spatial position, which allows a role for low-level forward and backward visual masking in RD (see MacKay, Abrams, Pedroza, \& Miller, 1996). However, we can think of no way that reduced forward and backward visual masking in the present paradigm might explain the different pattern of age effects in the two studies.

In lieu of a procedurally based account, theoretical details of how the repeated elements interact to cause $\mathrm{RD}$ in the two paradigms warrant further examination. The binding hypothesis for RD in RSVP sentences postulates an interaction within the system for forming new connections, such that two new connections cannot be rapidly formed to link one and the same node representing a repeated word to the two different nodes representing the phrases containing the two repetitions of the word. However, a binding RD hypothesis for the present paradigm must postulate interactions between different sorts of processes: activation of the node for the pretarget letter (e.g., the initial $i$ in hospitiable in its familiar position in the word), reactivation of that same node for the (identical) target letter (i.e., the added $i$ in hospitiable), and the mechanism for forming the new connection to represent the novel position of that target letter in the word. Within this more detailed theoretical analysis, aging may affect the rapid formation of two new connections from one and the same node, without affecting the interaction that causes "binding RD" in the present paradigm (i.e., reactivation of a highly practiced unit within a brief period of time, followed by new connection formation to represent the novel sequential position of that target unit). Another possibility is that aging may affect formation of new connections involving phrases in unfamiliar sentences and word lists (see MacKay \& Abrams, 1994), but not formation of new connections involving orthographic or phonological units.

\section{The Refractory Period RD Hypothesis}

Slowing of a response to a stimulus due to a prior response to the same stimulus is a type of refractory period that is relatively longer for older than young adults (see Welford, 1977). If this type of refractory period causes $\mathrm{RD}$, then $\mathrm{RD}$ should interact with age, repetition, and presentation rate in retrieval, such that older adults experience more $\mathrm{RD}$ than young adults at moderate rates but equivalent $R D$ at fast and at slow rates (see MacKay et al., 1994, for a detailed illustration and discussion of these predictions). Our results failed to support these predictions. A 2 (age) $\times 2$ (repetition) $\times 3$ (rate, a three-level factor for young and older adults) multivariate analysis of variance (MANOVA) on the detection data did yield an Age $\times$

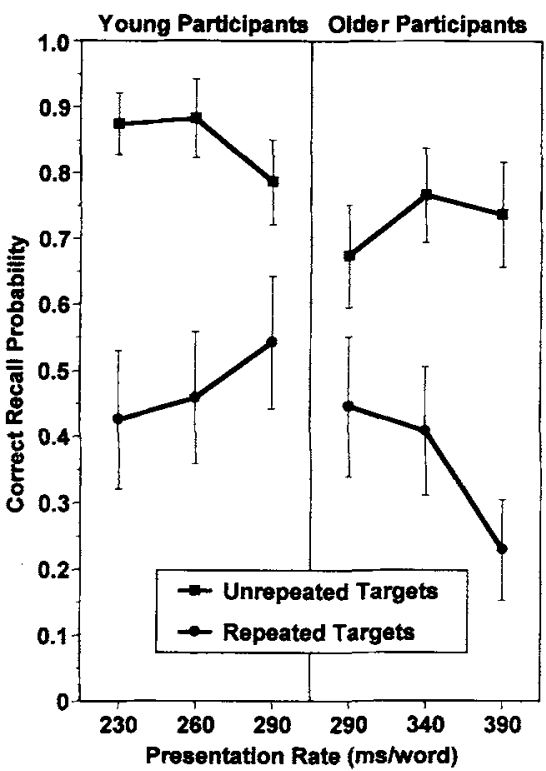

Figure A1. Double conditional probability (given correct detection and pretarget recall) of correctly recalling repeated- versus unrepeated-letter targets as a function of presentation rate for young and older adults.

Repetition $\times$ Rate interaction $(p<.046)$, but not the interaction predicted under the refractory period hypothesis (i.e., performance was poorer for the middle rate than either the faster or slower rates only for the young participants, and only for repeated-letter misspellings). No other interactions involving age or rate in these detection data approached significance. Also, the predicted Age $\times$ Rate interactions did not appear in double conditional analyses of retrieval. Figure A1 shows the double conditional probability of correctly retrieving repeated-and unrepeated-letter targets as a function of presentation rate for young and older adults, and a 2 (age) $\times 2$ (repetition) $\times 3$ (rate: fast, medium, and slow) MANOVA on these data (excluding 16 young and 20 older adults with empty data cells due to double conditionalization) revealed a main effect of age, $F(1,34)=5.01$, $M S E=0.15, p<.032$, and of repetition, $F(1,34)=66.21, M S E=0.11$, $p<.001$, but no main effect of rate $(F<1)$. In addition, although the Age $\times$ Repetition $\times$ Rate interaction approached significance, $F(2,68)$

${ }^{\text {A2 }}$ Simultaneous presentation raises the issue of whether milliseconds per letter or milliseconds per word is the most appropriate descriptor for comparing effects of rate in the present study versus other studies. Measured in milliseconds per word, the present $230-$ to $390-\mathrm{ms} /$ word rates are slower than in any previous demonstration of repetition deficit (RD): MacKay, Miller, and Schuster (1994) showed that young and older adults no longer exhibited RD in the standard rapid serial visual presentation paradigm at rates slower than $210 \mathrm{~ms} /$ word. However, measured in milliseconds per letter, presentation rates (about $27-46 \mathrm{~ms} / \mathrm{letter}$ ) resemble rates for previous demonstrations of RD (about $27-60 \mathrm{~ms} / \mathrm{phoneme}$ ).

${ }^{\mathrm{A} 3}$ Close inspection of Figure A1 also illustrates the need for adopting a wide range of rates in assessing the overall effects of aging on repetition deficit (RD): Maximal RD occurred at one rate for young adults (230 $\mathrm{ms} /$ word) and at a different rate for older adults ( $390 \mathrm{~ms} /$ word). In addition, although minimal RD may have occurred at the same rate $(290 \mathrm{~ms} /$ word $)$ for young and older aduits, retrieval $R D$ at $290 \mathrm{~ms} /$ word was decreasing with rate for young adults and increasing with rate for older adults (see Figure A1). These trends suggest that RD could be equivalent, less, or greater for young than older adults at any single, arbitrarily chosen rate. 
$=2.79, M S E=0.10, p<.069$, there were no Rate $\times$ Repetition, Age $\times$ Rate, or Age $\times$ Repetition interactions $(F s<1)$. ${ }^{\mathrm{A} .3}$

To check whether the extensive data discard in Figure A1 worked against the Age $\times$ Rate interactions predicted under the refractory period RD hypothesis, we conducted similar analyses for single conditional data (correct target recall given correct pretarget recall). A 2 (age) $\times 2$ (repetition) $\times 3$ (rate: fast, medium, and slow) MANOVA on these data indicated a main effect of age, $F(1,67)=7.67, M S E=0.14, p<.007$, with young adults correctly reproducing target letters more often than older adults, and a main effect of repetition, $F(1,67)=264.46, M S E=0.09, p<$ .001 , with unrepeated targets reproduced more often than repeated targets. However, neither the Age $\times$ Rate nor Age $\times$ Rate $\times$ Repetition interaction was reliable (smallest $p=.21$ ). These data therefore fail to support the refractory period RD hypothesis. ${ }^{\mathrm{A} 4}$

The present results also failed to support the predicted quadratic relations among $\mathrm{RD}$, presentation rate, and age, with deviations from linearity in reproducing repeated targets appearing at a faster rate for young than older adults. For the single conditional data, polynomial trend analyses of $\mathrm{RD}$ as a function of rate gave zero-order intercept models as the best fit outcome for both young and older adults, with predicted population means (57.3\% for young adults, $41.1 \%$ for older adults) that remained constant over the three rates for both young adults $(p<.427)$ and older adults $(p<$ .231). Mean retrieval of unrepeated-letter targets $(75.5 \%$ for young adults, $56.9 \%$ for older adults) also remained constant over rate, with a zero-order intercept model as the best fit outcome for both age groups. Finally, retrieval of repeated-letter targets also remained constant over rate, with a zero-order intercept model as the best fit outcome for young adults $(p<.095)$, and older adults $(p<.243)$. Mean repeated-target retrieval (17.8\% for young adults, $16.8 \%$ for older adults) was statistically indistinguishable for the two age groups in this model. In short, our data supported the background assumption or precondition of the refractory period $\mathrm{RD}$ hypothesis that retrieval is related to presentation time in the same way for young and older adults, but they failed to support predictions of this hypothesis: Retrieval of repeated targets varied linearly rather than quadratically with rate and age, with no Age $\times$ Rate interaction, no Repetition $\times$ Rate interaction, and no Age $\times$ Repetition $\times$ Rate interaction. Moreover, it seems unlikely that these interactions would have emerged given a wider range of rates. Our range of rates $(60 \mathrm{~ms}$ for young adults, $100 \mathrm{~ms}$ for older adults) was comparable to the range in MacKay et al. (1994; $60 \mathrm{~ms}$ for young adults, $130 \mathrm{~ms}$ for older adults) in which the main effect of rate and interactions involving rate were statistically signif- icant. Moreover, adopting faster rates seems likely to cause floor effects in older adults, and adopting slower rates seems likely to cause ceiling effects in young adults (see Figure A1), violating methodological preconditions for between-groups comparisons of RD (see the Method section and Mackay et al., 1994).

\section{Perceptual Fusion RD Hypothesis}

Perceptual fusion occurs when briefly presented repeated stimuli are perceived as fused, unrepeated, or no different from a continuous stimulus; according to Kline (1984), stimulus fusion (even for spatially separated stimuli) occurs over longer time periods for older adults because stimulus traces at all levels of representation persist longer in the aging nervous system. If perceptual fusion causes RD (as suggested, e.g., by Humphreys, Besner, \& Quinlan, 1988), so that repeated letters in our briefly presented stimuli are sometimes fused or represented as one in the mind, then Kline's age-linked fusion hypothesis predicts that older adults should fuse repeated letters over longer presentation times than young adults and that age, RD, and presentation rate should interact in the same way as in refractory period accounts of RD. Consequently, the results discussed above that contradict the refractory period RD hypothesis also contradict the perceptual fusion RD hypothesis. In this regard, it is of interest that results of Hochhaus and Marohn (1991, Experiment 4), MacKay et al. (1994), and MacKay and Miller (1996) also failed to support perceptual fusion accounts of RD. Occurrence of RD in the present retrieval task is also difficult to explain in a purely perceptual account such as the perceptual fusion RD hypothesis.

\footnotetext{
${ }^{\text {A4 }}$ In general, confidence in the meaningfulness of nonsignificant and significant effects reported in the present article is warranted because a priori, our design provided sufficient power to detect moderate-to-large size effects, but not so much power as to detect small size effects (see Levin, 1997, for the rationale). For example, power computed using Woodward, Bonett, and Brecht (1990) was .82 at the .001 level for detecting medium effect sizes such as the main effect of age in Figure A1, but power was .36 at the .05 level for detecting small size effects such as the small Age $\times$ Rate $\times$ Repetition effect in these same data.
}

Received April 23, 1997

Revision received May 22, 1998

Accepted May 22, 1998 